\title{
Restriction Fragment Length Polymorphism (RFLP)
}

National Human Genome Research Institute (NHGRI)

\section{Source}

National Human Genome Research Institute (NHGRI). Restriction Fragment Length

Polymorphism (RFLP).

Restriction fragment length polymorphism (RFLP) is a type of polymorphism that results from variation in the DNA sequence recognized by restriction enzymes. These are bacterial enzymes used by scientists to cut DNA molecules at known locations. RFLPs (pronounced "rif lips") are used as markers on genetic maps. Typically, gel electrophoresis is used to visualize RFLPS. 